\title{
Effects of Electrode Configuration and Stimulus Level on Rate and Level Discrimination with Cochlear Implants
}

\author{
Deborah J. Morris and Bryan E. Pfingst \\ Kresge H earing Research In stitute, Department of Otolaryngology, University of M ichigan, Ann Arbor, MI 48109-0506, USA
}

Received: 27 January 2000; Accepted: 27 July 2000; O nline publication: 17 O ctober 2000

\begin{abstract}
Recent studies have demonstrated that speech perception with cochlear implants can be significantly affected by electrode configuration. Contrary to expectations, broader configurations (monopolar or broad bipolar) produced equal or better speech recognition compared with narrower configurations (narrow bipolar or common ground). O ne hypothesis that would account for these results is that broader configurations excite larger populations of neurons providing a more robust representation of information on each channel of the prosthesis. It is known that the number of neurons excited by an electrical stimulus increases considerably as the stimulus level increases. Furthermore, many types of discrimination improve as a function of stimulus level. If the discrimination improvements seen with increasing stimulus level are due to increasing the size of the neural population carrying the signal, and if broadening the electrode configuration also increases the size of the activated neural population, then one would expect level and electrode configuration to affect discrimination in similar ways. To test this hypothesis, we studied several types of discrimination as a function of level and electrode configuration in four nonhuman primates with cochlear implants. We tested electrode configurations that produced current fields ranging from very restricted (tripolar) to broad (parallel monopolar). For each configuration, pulse-rate discrimination, amplitudemodulation-frequency discrimination, and level discrimination were tested at current levels spanning
\end{abstract}

Correspondence to: Bryan E. Pfingst, Ph.D. - Kresge H earing Research Institute - 1301 East Ann Street - Ann Arbor, MI 48109-0506. Telephone: (734) 763-2292; fax: (734) 764-0014; email: bpfingst@umich.edu much of the psychophysical dynamic range. Results showed large effects of current level on discrimination in many cases. H owever, effects of electrode configuration at comparable levels within the dynamic range were smaller or absent. Furthermore, the effect of level on discrimination was independent of electrode configuration in most cases even though the rate of spread of neural activation with level is expected to depend on electrode configuration. Possible interpretations of these results are that (1) the current level adjustments necessar y to achieve comparable loudness for the various configurations significantly countered any effects of electrode configuration on the size of the activated neural population, or (2) the effects of level on discrimination do not result from its effects on the spatial extent of neural activation.

Keywords: cochlear implants, electrode configuration, frequency discrimination, intensity discrimination, nonhuman primates, stimulus level

\section{INTRODUCTION}

Cochlear implants provide hearing to deaf patients by stimulating the auditory nerve with electrical signals that represent features of the patients' auditory environment. These prostheses use processors that divide the environmental acoustic signal into components and send each component through a separate channel to a distinct set of electrodes implanted in the cochlea. Recently, several studies have demonstrated that the configuration of the electrodes stimulated by individual channels of the prosthesis can have significant effects on the subjects' speech recognition and on the subjective quality of the sound evoked by electrical stimulation (Lehnhardt et al. 1992; Zwolan et al. 1996; Pfingst et al. 1997). The objective of the present study 
was to better understand the effects these electrode configurations have on perception.

For many years, it was assumed that narrow electode configurations, such as narrowly spaced bipolar configurations, would be advantageous for speech recognition because they would allow for better separation of the neural populations carrying information presented by the various channels of the processor. This theory was based on two assumptions: (1) that narrow electrode configurations (e.g., narrow bipolar or tripolar configurations) excite more spatially restricted arrays of neurons than broad configurations (e.g., broad bipolar or monopolar) and (2) that more spatially restricted excitation patterns would be an advantage for speech perception because they would reduce channel overlap. These assumptions, however, have been challenged by experiments (Lehnhardt et al. 1992; Zwolan et al. 1996; Pfingst et al. 1997) that show equal or better speech recognition in some patients with broad configurations compared with more narrow configurations. These data suggest several alternative hypotheses. Among them is the hypothesis that broad excitation patterns convey more information per channel because there are more neurons implementing the neural codes.

The studies of effects of electrode configuration on speech recognition are complicated by the complex nature of the speech signal, as well as features of the auditory prosthesis speech processors. Therefore, to help understand the nature of the effects of electrode configuration, we and others are conducting a series of experiments of a simpler nature. Previous studies and models have focused on effects of electrode configuration on stimulus detection, particularly on psychophysical strength-duration functions (Colombo and Parkins 1987; Pfingst et al. 1995a, 1995b; Smith and Finley 1997; Bruce et al. 1999). In the experiments reported here, we examined discrimination of simple signals presented at a single location in the electrode array (a single channel). Pulse-rate discrimination, amplitude-modulation-frequency discrimination, and level discrimination were tested as a function of level using several electrode configurations ranging from narrow to broad. Discrimination vs. level functions were obtained in order to help understand the nature of any differences observed as a function of electrode configuration. It is important to look at the data in this way because stimulus level can have strong effects on discrimination of electrical signals, and because the level of current required to achieve a comfortable loudness for discrimination testing varies considerably as a function of electrode configuration. Thus, we compared the effects of electrode configuration on discrimination at matched current levels, at matched sensation levels ( $d B$ re the detection thresholds), and at matched percentages of the dynamic range.
Stimulus level is known to affect the number of neuronsexcited by each channel of the prosthesis. The number of neurons activated increases dramatically as stimulus level is increased above the threshold of the most sensitive neurons (Snyder et al. 1990; Kral et al. 1998). Also, as noted above, increases in stimulus level result in improvement in some types of discrimination. Sinusoidal frequency discrimination (Pfingst and Rai 1990), pulse-rate discrimination (Pfingst et al. 1994), level (intensity) discrimination (Pfingst et al. 1983; Shannon 1983), and detection of amplitude modulation (Shannon 1992) all improve as a function of stimulus level. Electrode-place discrimination improves as a function of level in some cases, but these effects are smaller and less reliable than the effects of level on the temporal and level difference limens (Pfingst et al. 1999). We reasoned that (1) if the effects of stimulus level on temporal and level discrimination were due to stimulation of increased numbers of neurons at higher levels and (2) if broader electrode configurations excited larger numbers of neurons than narrow configurations, then broader configurations should produce better discrimination.

The studies described here were done using nonhuman primate subjects with multielectrode cochlear implants and percutaneous electrical connectors. The use of a percutaneous connector allowed for the use of a greater variety of electrode configurations than is available in most of the commercial cochlear implants that are used in humans. Some of these configurations used multiple scala tympani electrodes, each referenced to an extracochlear ground, and the size of the current field was controlled by adjusting the relative amplitudes and phases of the currents delivered to each of the electrodes (Pfingst et al. 1995a; Spelman et al. 1995). The narrowest configuration tested was a tripole (sometimes called a quadrapole), which produces a current field that is narrower and more symmetric than a typical narrow bipole (Jolly et al. 1996; $\mathrm{Kral}$ et al. 1998). The broadest configuration was a parallel monopolar configuration that produces a current field wider than a simple monopole. Longitudinal bipolar configurations with various electrode separations and simple monopolar configurations were also tested.

\section{METHODS}

Subjects, training, and housing facilities

The subjects were four adult male macaques ( two $M$. mulatta and two $M$. radiata) ranging in age from 10 to 21 years at the time of testing. Prior to deafening and implantation, subjects were trained using acoustic stimuli. Positive-reinforcement operant-conditioning 


\begin{tabular}{|c|c|c|c|}
\hline \multicolumn{4}{|c|}{ Subject implant specifications } \\
\hline Subject & $\begin{array}{l}\text { Estimated insertion depth of stimulated electrodes } \\
\text { ( } \mathrm{mm} \text { past the round window niche) }\end{array}$ & $\begin{array}{l}\text { Adjacent electrode } \\
\text { separation }(\mathrm{mm})\end{array}$ & $\begin{array}{l}\text { Electrode } \\
\text { width }(\mathrm{mm}\end{array}$ \\
\hline AF-R-1 & $\mathrm{B}=4.65, \mathrm{C}=3.90, \mathrm{D}=3.15, \mathrm{E}=2.40, \mathrm{~F}=1.65$ & 0.75 & 0.2 \\
\hline BE-L-1 & $\mathrm{A}=4.5, \mathrm{~B}=3.0, \mathrm{C}=1.6$ & $1.4-1.5$ & $\sim 0.3$ \\
\hline $\mathrm{HO}-\mathrm{R}-2$ & $\mathrm{~A}=1.7, \mathrm{~B}=1.0, \mathrm{C}=0.5$ & $0.5-0.7$ & $\sim 0.3$ \\
\hline NI-R-2 & $\mathrm{C}=1.0, \mathrm{D}=0.7$ (implant buckled upon insertion) & 0.6 & 0.2 \\
\hline
\end{tabular}

procedures were used to train subjects to perform psychophysical tasks that could be used to measure absolute detection thresholds, dynamic ranges, and various difference limens. During training and testing sessions, the subjects sat in custom-made primate chairs in double-walled sound-attenuating chambers (IAC type 1201A or Tracoustics model RE-240-B). Positioned in front of the subject was a light-display panel to signal the animal when the session began, a telegraph key within reach of either hand to record the animal's response, and a tube for delivery of an applesauce reinforcement when a correct response was made. Between these test sessions, the subjects were housed in individual cages in facilities accredited by the American Association for Laboratory Animal Care. In this home cage, the animals had continuous access to water and were fed once a day following the test session, and the environment was enriched with a variety of toys, mirrors, and fruit.

Implants, surgical procedures, and postsurgical stabilization

Upon completion of training, subjects were deafened in one ear with an intercochlear injection of neomycin and had a multiple-electrode array implanted in the scala tympani. Surgery was performed under isoflurane anesthesia using standard sterile operating procedures. After a subject was prepared and draped, the middle ear was approached by enlarging the external meatus. The round window membrane was penetrated with a 30 gauge needle and $50 \mu \mathrm{l}$ of a $10 \%(w / v)$ neomycin sulfate solution was slowly injected into the scala tympani. The round window membrane was then removed and the scala tympani electrode array was inserted through the round window into the scala tympani. Wires from all electrodes were led under the skin and muscle to a percutaneous connector mounted on the skull (Pfingst et al. 1989). A remote ground electrode was implanted under the temporalis muscle or, in one case (BE-L-1), under the bone defect of the implanted ear.

Three out of the four subjects were implanted with a scala tympani Type II implant (Xue and Pfingst 1989) built in our facilities. These consisted of 6 band electrodes surrounding a silicone rubber carrier. All 6 bands were inserted into the scala tympani when possible (subject NI). Because of surgical restraints, only the 3 most apical electrodes were inserted in subjects $B E$ and $H O$. The remaining subject $(A F)$ was implanted with a scala tympani implant constructed by Nucleus, Ltd. (Sydney, Australia) that consisted of 11 band electrodes, 8 of which were inserted into the scala tympani and 3 of which were left outside the cochleostomy. For subject AF, only 5 of the 8 implanted electrodes ( $B$ through F) were used for this study. In general, electrode separations varied from 0.5 to 1.5 $\mathrm{mm}$ (center to center). Subject-by-subject implant specifications (insertion depths, electrode separations, and electrode band widths) are listed in Table 1.

I mplants were identified by three letters and a number (e.g., see Fig. 1). The first two letters indicated the first two letters of the subject's name and the third letter indicated which ear was implanted. The number indicated how may times that ear had been implanted, i.e., 1 is the first implant in that ear, and 2 is the second implant in that same ear following removal of the first implant. Electrodes were labeled in al phabetical order from apex to base, i.e., A was the most apical electrode. In all cases, $G$ indicated the remote ground electrode.

Following implantation, thresholds for electrical stimuli were measured as a function of time. Thresholds were measured until stable (standard deviation $\leq 1 \mathrm{~dB}$ for the last 5 thresholds). Stabilization of the thresholds occurred approximately between 10 and 80 days after surgery. As in previous studies, reimplanted earstended to stabilize more quickly than firsttime implanted ears. Once the thresholds were stable, a variety of psychophysical data, including those reported in this article, were collected.

\section{Electrode configurations}

A variety of electrode configurations were tested. These fell into four categories: longitudinal tripolar, longitudinal bipolar, single monopolar, and parallel monopolar. For descriptive purposes, we designate "active" and "return" electrodes in each electrode 


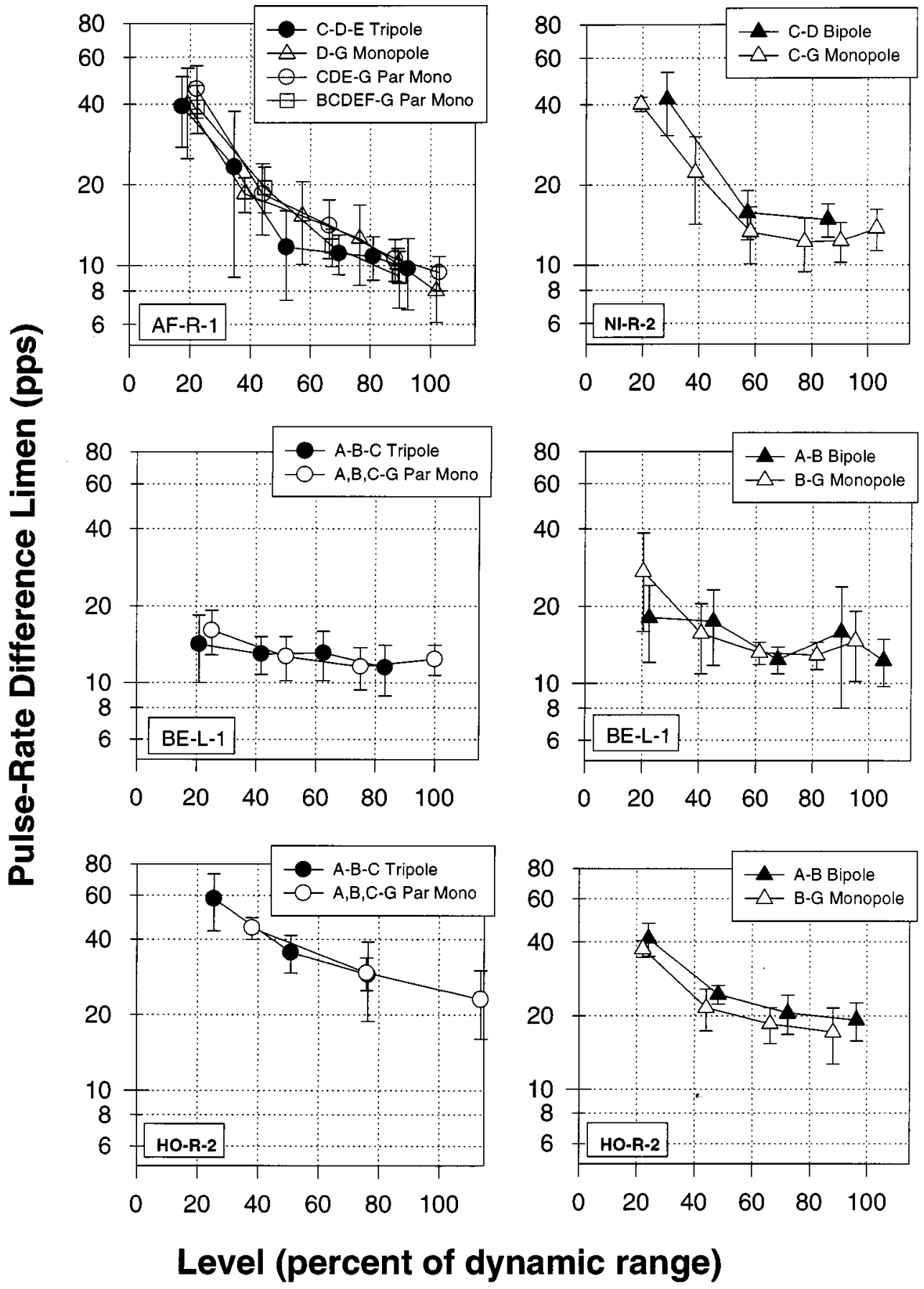

FIG. 1. Pulse-rate difference limens plotted as a function of level (percent of dynamic range) for six cases for various electrode configurations. Subject and implant identifications are indicated in the lower-left corner of each graph, and electrode configurations are indicated in the legends in the upper-right corners. For each case, only configurations that were tested during the same time period are compared. For subjects $\mathrm{BE}$ and $\mathrm{HO}$, two different pairs of configurations were tested during different time periods so the data for these subjects are shown in two separate panels. Each data point represents the mean plus and minus one standard deviation for 10 repeated difference limen estimates, each based on 15-20 trials/stimulus. configuration. Longitudinal tripolar configurations comprise three adjacent electrodes arranged longitudinally in the electrode array. When stimulating in this mode, the two flanking electrodes each receive current at half the amplitude and opposite polarity to that delivered to the middle electrode. For the tripolar configuration, the center electrode is called the active and the two flanking electrodesare the returns. Longitudinal bipolar configurations comprise two electrodes arranged longitudinally. For the bipolar configuration, the more apical electrode in the pair is the active and the more basal electrode is the return. Single monopolar configurations comprise one intrascal ar electrode and one extracochlear electrode. Parallel monopolar configurations comprise multiple intrascalar electrodes and one extracochlear electrode. When stimulating in this mode, all the intrascalar electrodes receive current of the same amplitude and phase. For the monopolar and parallel monopolar configurations, the scala tympani electrodes are the active electrodes and the extracochlear electrode is the return. 


\begin{tabular}{|c|c|c|}
\hline \multicolumn{3}{|c|}{$\begin{array}{l}\text { TABLE } \mathbf{2} \\
\text { nental parame }\end{array}$} \\
\hline Experiment & Task & Reference stimuli \\
\hline 1 & Pulse-rate difference limens & $\begin{array}{l}\text { Pulse trains: } \\
0.5 \mathrm{~ms} / \mathrm{ph}, 100 \mathrm{pps}, 300 \mathrm{~ms} \text { duration }\end{array}$ \\
\hline 2 & Modulation-frequency difference limens & $\begin{array}{l}\text { Sinusoidally amplitude-modulated (SAM) pulse trains } \\
\text { Carrier: } \\
50 \mu / p h, 250 \mathrm{pps}, 500-\mathrm{ms} \text { duration pulse trains } \\
\text { Modulator: } \\
10-\mathrm{Hz} \text { sinusoids, } 50 \% \text { modulation depth }\end{array}$ \\
\hline 3 & Level difference limens & $\begin{array}{l}\text { Pulse trains: } \\
0.5 \mathrm{~ms} / \mathrm{ph}, 100 \mathrm{pps}, 300 \mathrm{~ms} \text { duration }\end{array}$ \\
\hline
\end{tabular}

\section{Experimental design}

Prior to beginning the first discrimination experiment, thresholds and dynamic ranges were measured for 0.5 $\mathrm{ms} /$ phase, 300-ms duration electrical pulse trains. Pulse rates from 100 to 170 pps in 5- or 10-pps steps were tested to determine if threshold levels were affected by pulse rate over the range of rates needed for the discrimination experiments. At least 5 thresholds were determined, in random order, at each pulse rate. There was no significant variation in the threshold as a function of pulse rate, so it was assumed that loudness did not change as a function of pulse rate within this range.

Thresholds were checked following each discrimination experiment to determine if they remained stable over time. If they were unstable, more thresholds were collected and these new values were used in the experiment that followed.

Once thresholds and dynamic ranges were determined, the three discrimination experiments listed in Table 2 were begun. Reference-stimulus levels were set at 3,6 , and $9 \mathrm{~dB}$ above threshold for all subjects. If subjects were not able to discriminate at these set levels in each experiment, reference-stimulus levels were raise to levels at which the subjects could discriminate. In subjects with dynamic ranges greater than $9 \mathrm{~dB}$, reference-stimulus levels were added to encompass the subject's entire dynamic range.

Since discrimination ability can change over time, with very long "learning" curves (Turner and Nelson 1982; Prosen et al. 1990), subjects were trained for two to three months until changes over time in difference limens were minimal. Data were then collected for selected sets of electrode configurations, with testing for all conditions randomized. Only data for conditions tested during a common time period were compared in order to avoid confounding effects of changes over time. In most cases, all configurations for a given subject were tested during the same time period. However, for subjects $\mathrm{HO}$ and $\mathrm{BE}$ for pulse-rate discrimination, we compared bipolar and monopolar con- figurations during one time period and tripolar with parallel monopolar stimulation during a separate time period. Therefore, these two data sets are treated as separate cases.

Psychophysical procedures

Detection thresholds and difference limens were measured using a go/no-go procedure. The subject depressed a telegraph key to initiate each trial, held the key during a randomly varied (1-6s) foreperiod (observing response), and released the key within $1 \mathrm{~s}$ (response period) following stimulus change. Stimuli were presented in bursts of 300 - or 500 -ms duration (see Table 2). The stimulus changed from the foreperiod signal to the response period signal during an interburst interval at the end of the foreperiod with no change in timing. For detection-threshold tasks, the stimulus change following the foreperiod was from inaudible ( $99 \mathrm{~dB}$ attenuation) to a level near the detection threshold. For discrimination tasks, the stimulus changed in pulse rate, amplitude-modulation frequency, or from one audible level to a higher level. Responses (releases within $1 \mathrm{~s}$ of stimulus change) were reinforced by delivery of $0.2 \mathrm{cc}$ of applesauce to a spout located near the subject's mouth.

Detection thresholds or difference limens were collected for one electrode configuration and referencestimulus level per session. Sessionswere run in random order until a predetermined number of detection thresholds or difference limens per reference-stimulus level had been collected for each electrode configuration. Each session consisted of a table of 10-12 stimuli varying in either level (from audible to inaudible) for detection threshold tasks or pulse rate, modulation frequency, or level (from easily discriminated to not discriminated) for discrimination tasks. Stimuli were presented by the method of constant stimuli. All stimuli in the table were run in random order until each totaled 15-20 trials (tables for which fewer than 15 trials/ stimulus were completed were not used). Percent responses were recorded for each stimulus in 
the table. Percent responses were then plotted as a function of stimulus level or frequency difference to form psychometric functions. Detection threshold or difference limen was defined as the point on the psychometric function (determined by linear interpolation) at which the subject responded on $50 \%$ of the trials.

Guess rates (releases during the 1-s unmarked observation period on trials where no stimulus change was presented) were measured during all sessions. We attempted to keep the guess rate low by controlling the duration of a penalty timeout, contingent on early releases. Stimulus levels and frequency ranges were selected so as to maintain a relatively constant rate of reinforcement across conditions in order to avoid conditions that might lead to a change in response strategy. With these methods, guess rates were usually kept within a range of $2 \%-20 \%$ and did not vary systematically across conditions. If these criteria for the guess rate were not met, the data were not used.

In order to determine each subject's dynamic range (used during the discrimination tasks), maximum comfortable current levels were measured during the threshold testing sessions. Maximum comfortable current levels were determined by observing reaction times while increasing the stimulus level. For each subject, a criterion reaction time was determined prior to deafening by recording the subject's median reaction time to loud (e.g., $98 \mathrm{~dB}$ SPL) acoustic noise. When the subject responded slightly faster than this criterion, continually released before the signal was presented, or refused to run at all at a high current level, the next lowest level was determined to be the upper limit of his dynamic range or the maximum comfortable current.

\section{Equipment and stimuli}

Pulsatile signals were generated by computer, stored in a stimulus register, and delivered under computer control. For all pulses, the leading phase to the active electrode was al ways negative. Sinusoidal stimuli were generated by a Rockland frequency synthesizer and gated by a toneswitch. Modulated signals were generated by amplitude modulating a pulse train with a sinusoid. The modulation depth was $50 \%$. Modulated signals were passed through a toneswitch with a risefall time of $100 \mathrm{~ms}$. Signalswere attenuated bya Wilsonics attenuator and then passed to the earspeaker (during training only) or to a controlled-current stimulator with capacitively coupled input and output modified from the design described by Spelman et al. (1978). The output of the stimulator was connected directly to the implants through the percutaneousconnector. Stimulus delivery and data collection were controlled by IBM PC computers using software developed locally.

\section{Statistical analyses}

Statistical analyses were performed only on cases that showed a consistent effect of electrode configuration. For these cases, we compared the relation ship between difference limen and level (in percent of dynamic range) for the different electrode configurationsusing analysis of covariance (ANCOVA). Analyses were performed on the log scale for pulse-rate and modulationfrequency difference limens, and on the linear- $d B$ scale for level difference limens. Linear and quadratic effects of level were tested, as well as interactions between electrode configuration and the linear and quadratic level effects. All analyses were performed using SAS statistical software (SAS Institute Inc., Cary, NC).

\section{Animal care assurance}

These studies were performed in accordance with the U nited States Public H ealth Ser vice Policy on H umane Care and Use of Laboratory Animals, the National Research Council Guidefor the Careand U seof $L$ aboratory Animals (1996), and the Animal Welfare Act (7U.S.C. et seq.). The animal-use protocol was approved by the University Committee on the $U$ se and Care of Animals at the U niversity of Michigan.

\section{RESULTS}

In general, the effects of stimulus level on the various difference limens that we measured were larger and more consistent than the effects of electrode configuration in the same animals. Difference limens decreased systematically as a function of level in many cases, while, in those same cases, electrode configuration had small effects or no effect. However, when electrode configuration did affect difference limens, the effect was in the expected direction. Configurations that are thought to produce broader spatial patterns of excitation tended to be associated with slightly smaller difference limens (better discrimination). Details of the results for each type of discrimination are given in the following sections.

Pulse-rate difference limens

The strongest effects of stimulus level were seen in the data for pulse-rate discrimination. Difference limens for pulse rates systematically decreased as a function of 


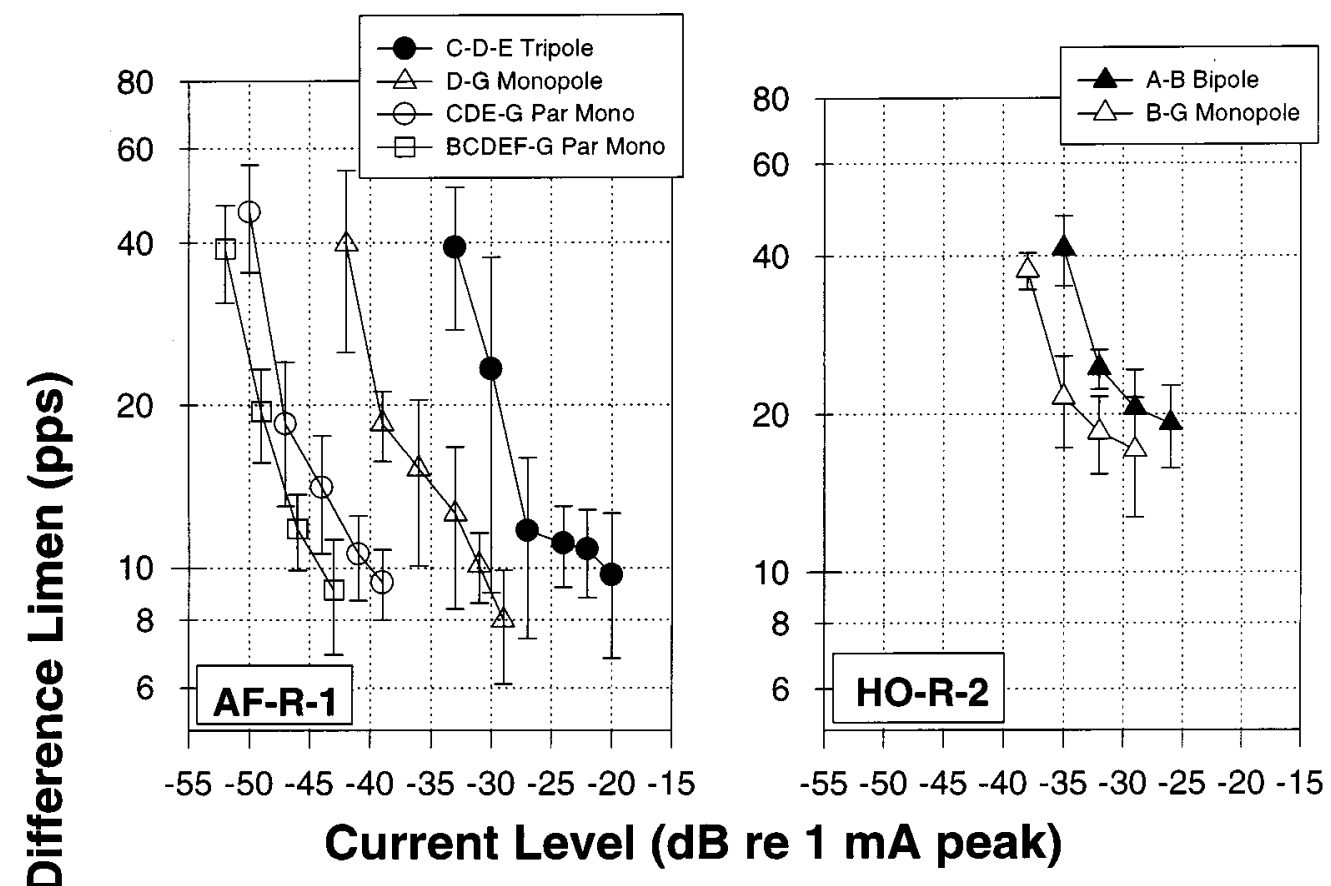

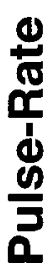

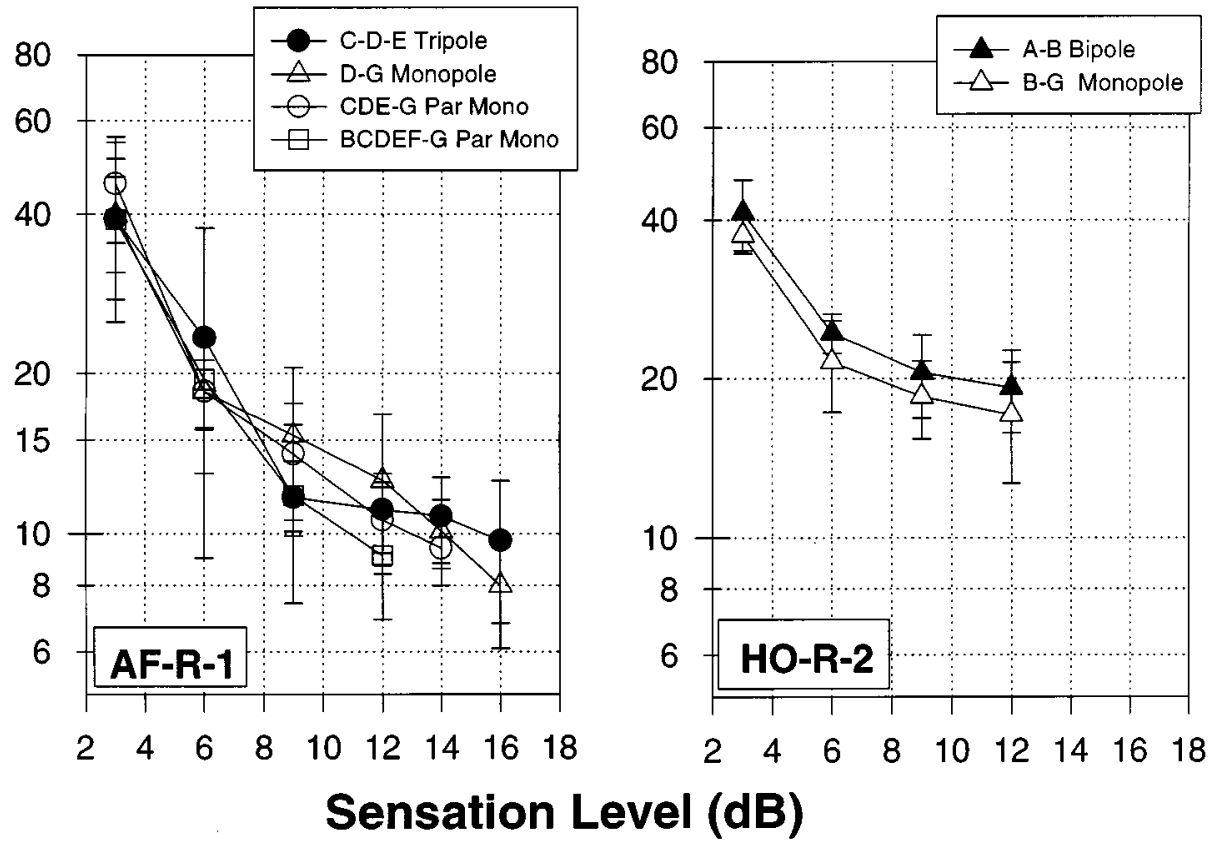

FIG. 2. Pulse-rate difference limens for two cases plotted as a function of stimulus level using two different scales for stimulus level: current in the upper half of the figure and sensation level ( $\mathrm{dB}$ re the detection threshold) in the lower half. Left column: Subject AF - 4 electrode configurations. Right column: Subject $\mathrm{HO}$ - bipolar vs. monopolar configurations. Each data point represents the mean plus and minus one standard deviation for 10 repeated difference limen estimates, each based on 15-20 trials/stimulus. stimulus level throughout most of the dynamic range. Figure 1 shows pulse-rate difference limens plotted as a function of level (in percent of dynamic range) for the six cases we tested. For three of the cases (AF and both cases for $\mathrm{HO}$ ), difference limens decreased systematically as a function of level throughout the dynamic range. For the remaining three cases ( $\mathrm{NI}$ and both cases for $\mathrm{BE}$ ), the highest difference limens were obtained at the lowest levels tested, but at the higher levels, threshold difference limens showed little change as a function of level.
In four cases (AF, HO - tripolar vs. parallel monopolar, and BE both cases), there wasno discernable difference in the difference limen vs. level (percent of dynamic range) functions obtained under the various electrode configurations (Figure 1 ). In the remaining two cases ( $\mathrm{HO}$ - bipolar vs. monopolar, and NI) difference limensfor the broader configuration were slightly lower than those for the narrower configuration. The ANCOVA was run for these two cases. It showed that level had a significant effect on the difference limen. A quadratic function (decreasing, then leveling off) 


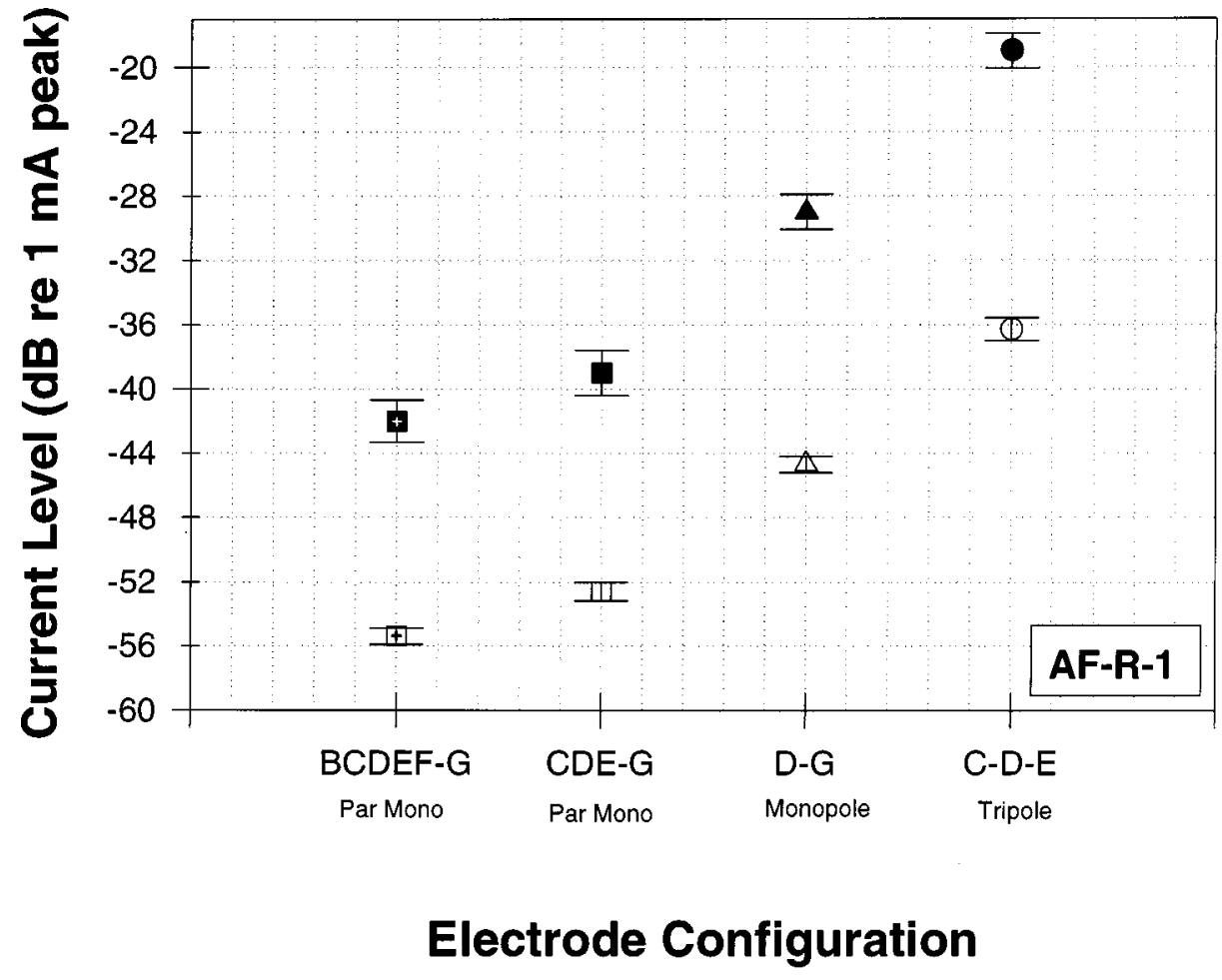

FIG. 3. Detection thresholds (open symbols) and maximum comfortable current levels (filled symbols) plotted as a function of electrode configuration for subject AF. Threshold did not differ as a function of pulse rate, so the mean threshold plus and minus one standard deviation for 8 pulse rates is shown for each electrode configuration. The configurations are arranged in order on the abscissa with the configuration presumed to produce the largest current field on the left and the configuration presumed to produce the most restricted current field on the right. However, placement on the abscissa is based only on presumed rank order, not on a quantitative estimate of field size. In the tripole, the $\mathrm{C}$ and $\mathrm{E}$ electrodes receive half the current in opposite polarity to that delivered to the $\mathrm{D}$ electrode. The two parallel monopolar electrode configurations (Par Mono) differ in number of electrodes. BCDEF-G has 5 scala tympani electrodes and CDE-G has 3 scala tympani electrodes. All scala tympani electrodes are $0.75 \mathrm{~mm}$ center to center. $G$ is a return electrode under the temporalis muscle. provided a significantly better fit than a linear function $(\mathrm{F}=87.65 ; \mathrm{df}=1,86 ; \mathrm{p}=0.0001$ for $\mathrm{NI}$ and $\mathrm{P}=$ 89.21; $d f=1,76 ; p=0.0001$ for $\mathrm{HO}$ ). The electrodeconfiguration effect was also significant $(F=20.60$; $\mathrm{df}=1,86 ; \mathrm{p}=0.0001$ for $\mathrm{NI}$ and $\mathrm{F}=14.94 ; \mathrm{df}=1$, $76 p=0.0002$ for $\mathrm{HO}$ ), with the difference limens for the monopolar electrode configuration being smaller than the difference limens for the bipolar. No interaction terms were significant for either case, indicating that the functional relationships between difference limens and level did not change with electrode configuration, other than raising or lowering.

Since electrode configuration affects the level of current required for stimulus detection and the dynamic range from detection threshold to maximum comfortable level, and since stimulus level affects pulse-rate difference limens, care must be taken in interpreting the effects of electrode configuration. For this reason, we examined the pulse-rate discrimination versus level functions using three different abscissas for stimulus level. Examples are illustrated in Figure 2, which shows the data for two cases plotted as a function of current level (top row) and sensation level (bottom row). The data for these two cases, plotted as a function of percent of dynamic range, are shown in Figure 1.

As expected, the currents required for stimulation within the dynamic range of hearing varied significantly as a function of electrode configuration, being lowest for the broadest configurations. Detection thresholds and maximum comfortable current levels for subject AF are plotted as a function of electrode configuration in Figure 3. Since the currents used for the discrimination tasks were selected to be between the threshold and maximum comfortable loudness levels, the functions in the upper row of Figure 2 (pulserate difference limens vs. current level in $\mathrm{dB}$ re $1 \mathrm{~mA}$ peak) are located at different regions along the abscissa. The functions for the broader configurations (parallel monopolar and monopolar) are located to the left of the graph while those for narrower configurations (bipolar and tripolar) are located to the right. At any given current level, difference limens for the narrower configurations are higher, provided the signal is audible. However, this is not a very meaningful comparison, because testing at the same current level for the different configurations would be at markedly different loudness levels. When the difference limens are plotted in terms of sensation level, the functions are seen to be very similar. In a few cases, the difference limens for the broader configuration were lower, as in subject $\mathrm{HO}$ in Figure 2, the lower right example.

Note that in cases where the discrimination for the broader electrode configuration was slightly lower than that for the narrower configuration, increasing the stimulus level for the narrower configuration by only a few $\mathrm{dB}$ (in the difference limen vs. sensation level functions in Fig. 2) or a few percent of the 

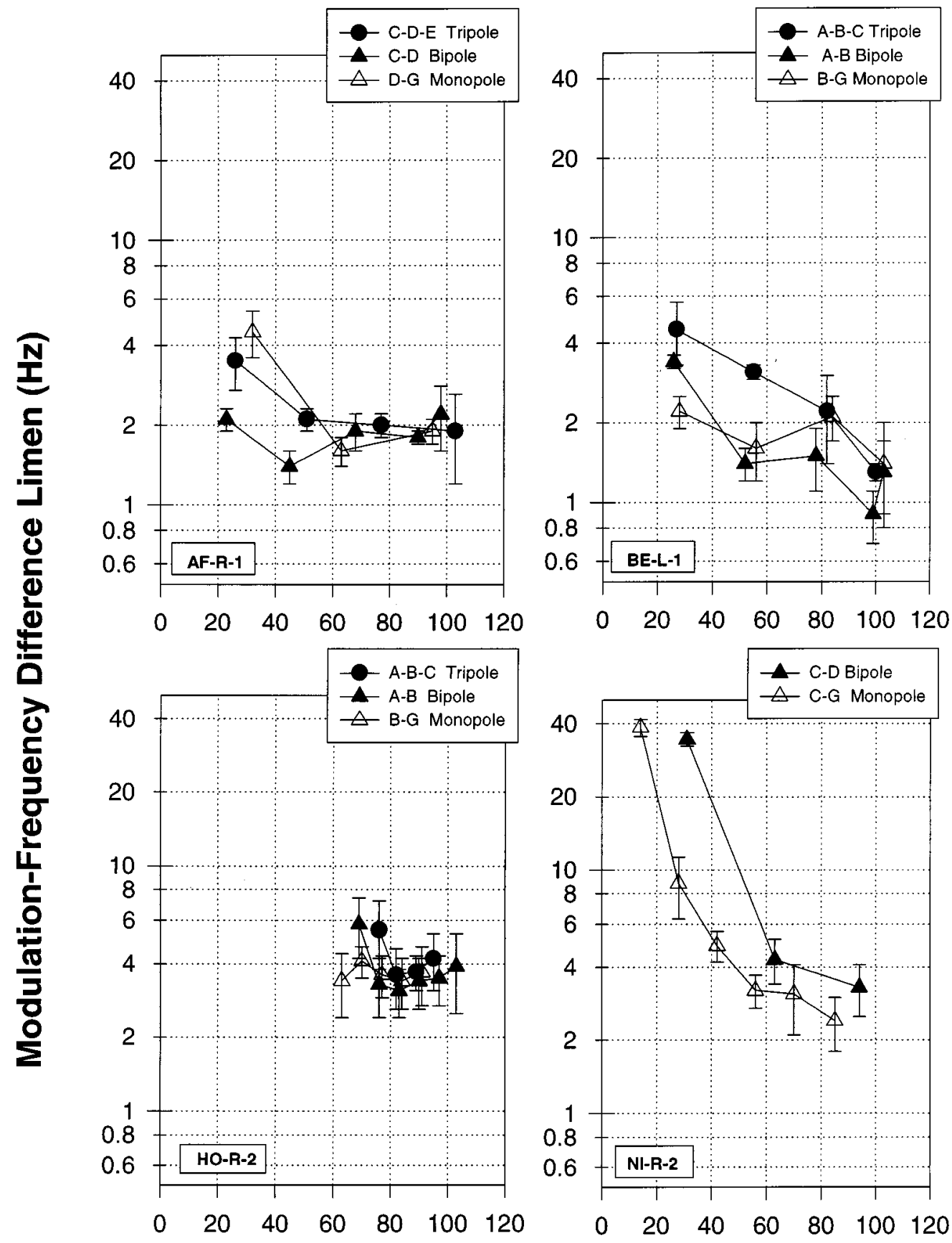

Level (percent of dynamic range)

FIG. 4. Modulation-frequency difference limens plotted as a function of level (in percent of dynamic range) for four subjects for various electrode configurations. All electrode configurations shown for a given subject were tested during the same time period in random order. Each data point represents the mean plus and minus one standard deviation for $3-5$ repeated difference limen estimates, each based on 15-20 trials/stimulus.

dynamic range (in the functions shown in Fig. 1) would result in discrimination for the two configurations being equal, at least at the lower levels. This is true as long as the slope of the difference limen vs. level function remains negative. In regions where the slope is near zero (e.g., Fig. 1 , subject $\mathrm{NI}$ at $>60 \%$ of the dynamic range), the difference limens for the broader configuration would presumably remain slightly lower than those for the narrower configuration even if the level of the narrower configuration were increased by several $\mathrm{dB}$.
Modulation-frequency difference limens

Modulation-frequency difference limens plotted as a function of level (in percent of dynamic range) for various electrode configurations are shown in Figure 4. Results are similar in several ways to those obtained in the pulse-rate discrimination experiment. Two subjects (BE and $\mathrm{NI}$ ) showed decreases in modulationfrequency difference limens throughout the dynamic range, while one subject (AF) showed decreases as a function of level in the lower half of the dynamic range 


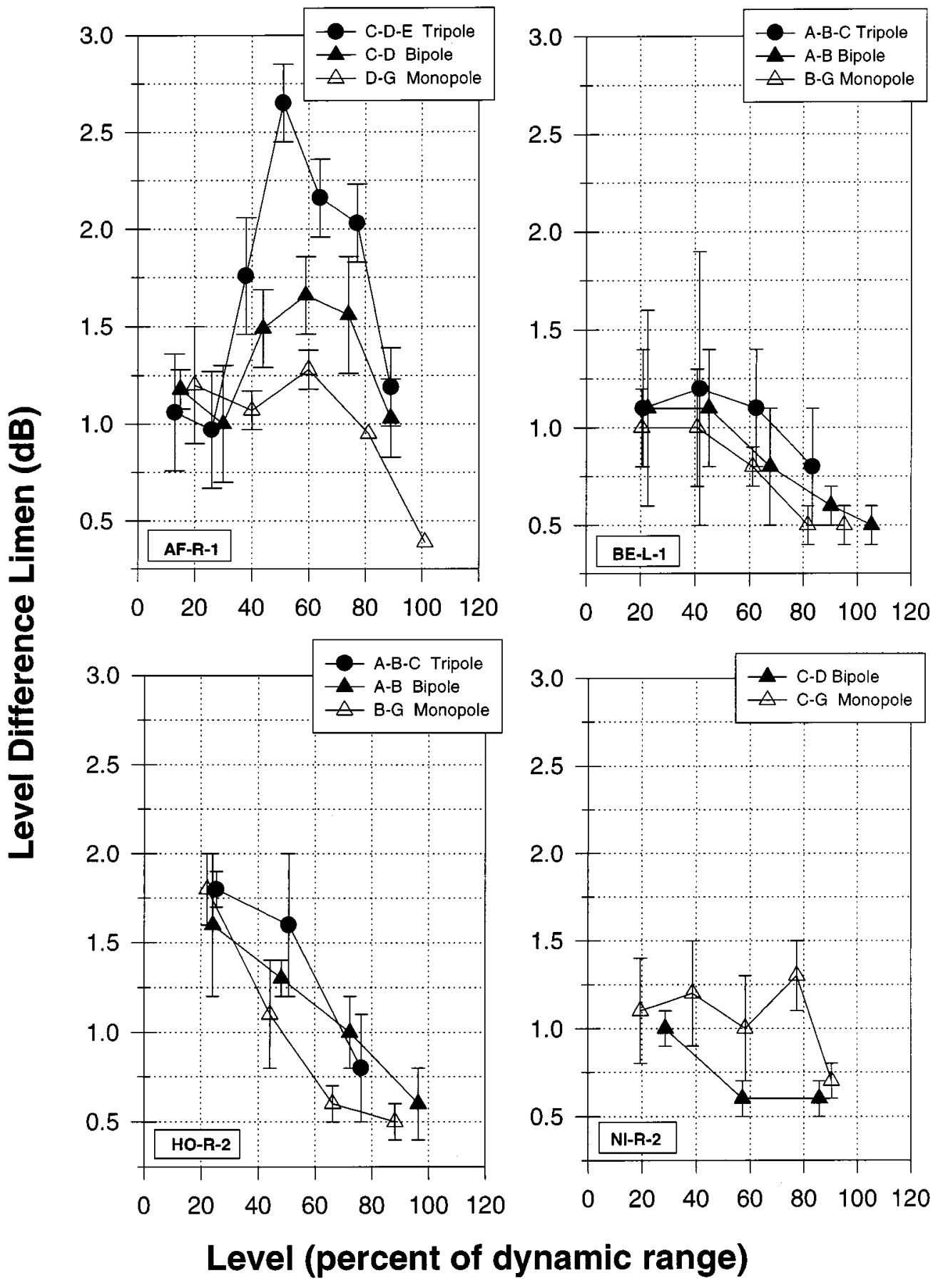

FIG. 5. Level difference limens plotted as a function of level (in percent of dynamic range) for four subjects for various electrode configurations. All electrode configurations shown for a given subject were tested during the same time period in random order. Each data point represents the mean plus and minus one standard deviation for 5 repeated difference limen estimates, each based on 15-20 trials/stimulus. but little change as a function of level at higher levels. Note, however, that the pattern of change in the modulation-frequency difference limens across level was not the same within subjects as that observed in the pulserate discrimination experiment. For example, while subject AF showed decreases in pulse-rate difference limens as a function of level throughout the dynamic range, he showed decreases in modulation-frequency difference limens only in the lower half of the dynamic range. Subject $\mathrm{HO}$ was not tested at the lower half of the dynamic range because he would not respond in this task at the low levels. We found consistent differences in the functions based on electrode configuration in only one subject (NI). Here the difference limens for the broader configuration were lower than those for the narrower configuration. For this case, the ANCOVA again showed a significant quadratic effect of level $(F=92.42 ; d f=1,40 ; p=0.0001)$ and $a$ significant electrode-configuration effect $(F=94.07$; $\mathrm{df}=1,40 ; p=0.0001$ ), with the difference limens for the monopolar electrode configuration again being smaller than for the bipolar. In addition, a significant 
interaction between electrode configuration and level was found $(F=31.24 ; d f=1,40 ; p=0.0001)$, but no significant interaction between electrode configuration and the quadratic effect of level was found. These effects indicate that the curvature of the limen-level relationship was not affected by electrode configuration, but the curve for the bipolar electrode configuration was shifted slightly to the right, as well as being raised in comparison to the curve for the monopolar.

\section{Level difference limens}

Level difference limens plotted as a function of level (in percent of dynamic range) for various electrode configurations are shown in Figure 5. In most cases, difference limens decreased as a function of level over at least part of the dynamic range. The difference limens for subject AF were unusual in that the highest difference limens occurred in the middle of the dynamic range. All of the subjects except subject N I showed a systematic relationship between level difference limen and electrode configuration, with the configurations presumed to produce the broadest excitation patterns showing the lowest level difference limens. In general, increasing level over $80 \%$ of the dynamic range had a larger effect on level difference limensthan did changing the electrode configuration.

The level difference limen vs. level data (Fig. 5) were more complex than the temporal discrimination data. H owever, in all four cases, significant effects of electrode configuration were found ( $p=0.0280$ for $\mathrm{BE}$ and $p=0.0001$ for $\mathrm{AF}, \mathrm{HO}$, and $\mathrm{NI}$ ). In three cases, the electrode configurations were ordered with the monopolar having the lowest difference limens and the tripolar having the largest. However, in one case (NI), the monopolar configuration had significantly higher difference limens than bipolar. For cases $A F$, $\mathrm{HO}$, and $\mathrm{NI}$, significant interactions were observed between electrode configuration and the quadratic effect of level, indicating changes in the shape of the functional relationships among the electrode pairs $(p=0.0001$ for $A F, p=0.0027$ for $\mathrm{HO}$, and $p=0.0133$ for $\mathrm{NI}$ ). There were a variety of functional forms, however, as can readily be seen in Figure 5 . O nly the data for $\mathrm{HO}$ fit the model of a more rapid spread of excitation at lower levels for the monopole and least rapid spread at lower levels for the tripole.

\section{DISCUSSION}

The various electrode configurations used in thisstudy were intended to produce current fields of different sizes and, thus, to control the size of the activated neural population. If stimulation were presented by these various configurations using the same current level for each configuration, it is clear that the sizes of the current fields and the sizes of the activated neural populations would, in fact, vary systematically with electrode configuration. Of the configurations we tested, the smallest current fields would be generated by the tripolar configuration (Spelman et al. 1995; Jolly et al. 1996; Kral et al. 1998). Next in size would be the fields generated by the longitudinal bipolar configurations, which had electrode separations between 0.6 and $1.5 \mathrm{~mm}$. Monopolar configurations clearly produce larger current fields and activate larger populations of neurons than narrow bipolar configurations (Spelman et al.1995; Kral et al. 1998). The parallel monopoles would produce the largest current fields.

At the current levels at which the implants normally operate, however, the situation is more complicated. These currents must fall between the detection threshold level and the maximum comfortable loudness level; these levels vary considerably as a function of electrode configuration (e.g., Fig. 3). Presumably, across the various configurations, the levels that produce equally loud sounds produce neural response patterns that are equal in some way, but it is not known if these response patterns are equal in spatial distribution, total discharge rate, or some other neural response feature. It is clear that the differences in neural responses across the various electrode configurations will be smaller at equal-loudness levels than at equal-current levels because the narrower configurations require more current and because the size of the activated neural population increases as a function of current level. Again, the degree to which these differences in the stimulation current counteract the effects of electrode configuration on the size of the neural population is not known.

There is indirect evidence from psychophysical studies that the neural populations excited by broader electrode configurations are, in fact, broader than those excited by more narrow configurations, even at equal-loudness levels. One observation that is consistent with this picture is that the variation in threshold levels, or in maximum comfortable loudness levels, across the array of electrodes is consistently smaller for broad configurations than for narrow configurations (Pfingst et al. 1997). It is assumed that the lower variance for broader configurations occurs because the threshold or maximum comfortable loudness level results from integration across a larger population of neurons than is the case with narrow configurations. Thus, effects of local differences in the nerve population, and/ or other variables that give rise to the variance from electrode to electrode, when narrow configurations are used, are averaged out when broad configurations are used. 
A remaining unknown, however, is the magnitude of the effect of electrode configuration on the size of the neural population relative to the magnitude of the effect of stimulus level. The data from the current experiment suggest that the effects of electrode configuration on temporal discrimination are much smaller and less consistent than the effects of stimulus level. For level difference limens, the effects of electrode configuration are somewhat larger, but, in general, they are smaller than the effects of stimulus level. O ur original hypothesis was that stimulus level and electrode configuration should have similar effects on temporal and level difference limens because both would affect the size of the neural population carrying the information about the signal. In light of the present data, this hypothesis must be modified. O ne possibility is that the difference in the size of the neural population excited by narrow, asopposed to broad, configurations is much smaller than the difference in the size of the neural population excited by low-level versus high-level stimuli. Another possibility is that part of the effect of current level on discrimination is due to mechanisms other than the effects of level on the size of the neural population.

If discrimination acuity depends primarily on the number of neurons activated by the electrical stimulus, then we would expect an interaction between stimulus level and electrode configuration in their effects on temporal and level difference limens. This is because the rate of spread of neural excitation as a function of level isprobably greater for broad electrode configurations than for narrow configurations (e.g., see Snyder et al. 1990). Given these differences in the rate of spread of excitation as a function of level, we would expect to see that slopes of the difference-limen vs. level functions would be steeper for broad configurations than for narrow configurations. In contrast to this expectation, the data in the current study show approximately the same slopes regardless of electrode configuration, in most cases. Again, these data suggest either that the effects of electrode configuration on the spread of excitation with level are relatively small or that mechanisms unrelated to the number of fibers activated are primarily responsible for the effects of level on discrimination.

Whatever the mechanism by which electrode configuration affects discrimination, it may be that this also contributes in part to the effects of electrode configuration on speech recognition. As in the discrimination studies, the effects of electrode configuration on speech recognition are often small and occur in only some subjects (Zwolan et al. 1996; Pfingst et al. 1997). The reasons for variability of these effects has not been determined.

\section{ACKNOWLEDGMENTS}

This work was supported by NIH/ NIDCD grants DC00274 and DC03808. We express appreciation to Kevin Franck for his comments on the manuscript and to Kathy Welch and Brenda Gillespie of the University of Michigan Center for Statistical Consultation and Research for their input and statistical expertise.

\section{REFERENCES}

Bruce IC, White MW, Irlicht LS, O'Leary SJ, Clark GM. The effects of stochastic neural activity in a model predicting intensity perception with cochlear implants: Low-rate stimulation. IEEE Trans. Biomed. Eng. 46:1393-1404, 1999.

Colombo J, Parkins CW. A model of electrical excitation of the mammalian auditory-nerve neuron. H ear. Res. 31:287-311, 1987.

Jolly CN, SPelman FA, Clopton BM. Quadrupolar stimulation for cochlear prosthesis: modeling and experimental data. IEEE Trans. Biomed. Eng. 43:857-865, 1996.

Kral A, Hartmann R, Mortazavi D, Klinke R. Spatial resolution of cochlear implants: The electrical field and excitation of auditory afferents. Hear. Res. 121:11-28, 1998, DOI:10.1016/ S0378-5955, 98, 00061-6.

Lehnhardt E, Gnadeberg D, Battmer RD, von Wallenberg EL. Evaluation of different coding strategies and stimulation modes for the Nucleus multichannel cochlear implant. YANAGIHARA N, SUZUKI J, Transplants and Implants in Otology II Kugler Publications New York 1992, 283-290.

Paingst Be, Albrektsson T, Tjellstrom A, Miller JM, Zappia J, XUE XL, WeISER F. Chronic skull-anchored percutaneousimplants in non-human primates. J. Neurosci. Meth. 29:207-216, 1989.

PFingst BE, Burnett PA, SUtTon D. Intensity discrimination with cochlear implants. J. Acoust. Soc. Am. 73:1283-1292, 1983.

Pfingst Be, Holloway LA, Poopat N, Subramanya AR, Warren $M F$, ZwOLAN TA. Effects of stimuluslevel on nonspectral frequency discrimination by human subjects. Hear. Res. 78:197-209, 1994.

Pfingst BE, Holloway LA, Zwolan TA, Collins LM. Effects of stimulus level on electrode-place discrimination in human subjects with cochlear implants. Hear. Res. 134:105-115, 1999, DOI: 10.1016/ S0378-5955, (99), 00079-9.

Pfingst Be, Miller AL, Morris DJ, Zwolan ta, Spelman FA, CLOPTON BM. Effects of electrical current configuration on stimulus detection. Ann. Otol. Rhinol. Laryngol. 104 Suppl. 166:127131, 1995a.

Pfingst BE, Morris DJ, Miller AL. Effects of electrode configuration on threshold functions for electrical stimulation of the cochlea. Hear. Res. 85:76-84, 1995b, DOI:10.1016/0378-5955, (95), 00037-5.

PFINGST BE, RAI DT. Effects of level on nonspectral frequency difference limens for electrical and acoustic stimuli. Hear. Res. 50: 43-56, 1990.

PFingst BE, Zwolan TA, Holloway LA. Effects of stimulus configuration on psychophysical operating levels and on speech recognition with cochlear implants. Hear. Res. 112:247-260, 1997, DOI:10.1016/ S0378-5955, (97), 00122-6.

Prosen CA, Moody DB, Sommers MS, Stebins WC. Frequency discrimination in the monkey. J. Acoust. Soc. Am. 88:21522158, 1990.

SHANNON RV. Multichannel electrical stimulation of the auditory nerve in man. I. Basic psychophysics. Hear. Res. 11:157-189, 1983.

SHANNON RV. Temporal modulation transfer functions in patients with cochlear implants. J. Acoust. Soc. Am. 91:2156-2164, 1992. 
SMITH DW, FINLEY CC. Effects of electrode configuration on psychophysical strength-duration functions for single biphasic electrical stimuli in cats. J. Acoust. Soc. Am. 102:2228-2237, 1997, DOI:10.1121/ 1.419636.

Snyder RL, Rebscher SJ, Cao K, Leake PA, Kelly K. Chronic intracochlear electrical stimulation in the neonatally deafened cat. I. Expansion of central representation. Hear. Res. 50:7-33, 1990.

Spelman FA, Pfingst BE, Clopton BM, Jolly CN, Rodenhiser KL. The effects of electrical current configuration on potential fields in the electrically stimulated cochlea: Field models and measurements. Ann. Otol. Rhinol. Laryngol. 104 Suppl. 166:131-136, 1995.
Spelman FA, Pfingst BE, Miller JM. A constant-current stimulator for use with chronic cochlear implants. Proc. San. Diego Biomed. Symp. 17:1-3, 1978.

TURner CW, Nelson DA. Frequency discrimination in regions of normal and impaired sensitivity. J. Speech. Hear. Res. 25:34-41, 1982.

XUE X, PFIngSt BE. Inner ear implants for experimental electrical stimulation of auditory nerve arrays. J. Neurosci. Meth. 28:189196. 1989.

Zwolan TA, Kileny PR, Ashbaugh C, Telian SA. Patient performance with the Cochlear Corporation " $20+2$ " implant: Bipolar versus monopolar activation. Am. J. Otol. 17:717-723, 1996. 